\title{
Covid 19 y cirugía: ¿Qué hemos avanzado?
}

\section{Covid 19 and surgery: What have we advanced?}

El año 2020 ha marcado un antes y después en la historia de la humanidad. La pandemia de la COVID 19 ha significado la pérdida de gran cantidad de vidas humanas, ha expuesto las debilidades de nuestros sistemas de salud y junto con ello ahora asistimos a presenciar las secuelas que va dejando esta enfermedad.

Los médicos hemos tenido que afrontarla, cada uno aportando en el manejo de la misma de acuerdo con las competencias para las que ha sido formado. Los cirujanos no hemos estado ajenos a ella, todo lo contrario, hemos tenido que adaptarnos para realizar las labores que el contexto exige. La actividad quirúrgica no solo involucra el aspecto de labor asistencial dentro de hospitales públicos o privados; sino que está vinculada muy de cerca al rol docente, de investigación, actividad societaria y de gestión ${ }^{(1)}$.

En el Perú, los servicios quirúrgicos han tenido que reorganizarse. En el Hospital Cayetano Heredia, a inicios de la pandemia se conformó un sistema de comando de incidentes y se dieron disposiciones para proteger al personal vulnerable. Los cirujanos y residentes de cirugía que continuaron laborando, fueron asignados a la atención de pacientes con COVID neumológicos, y los servicios de hospitalización de cirugía fueron implementados para atender a pacientes con insuficiencia respiratoria. Los pacientes quirúrgicos electivos tuvieron acceso limitado a los hospitales para dar prioridad a la atención de los pacientes con la COVID 19. El Departamento de Cirugía generó sus propias guías de recomendaciones estableciendo flujogramas dinámicos para el adecuado triaje de los pacientes quirúrgicos de emergencia y urgencia; estos flujogramas han ido cambiando según la disponibilidad de pruebas diagnósticas y la evidencia generada de su adecuada utilización ${ }^{(1,2)}$.

Por otro lado, los procedimientos laparoscópicos, alternativa quirúrgica para el manejo de múltiples patologías abdominales, también fueron restringidos, principalmente por el temor a que los aerosoles generados por el neumoperitoneo en la insuflación y desuflación del abdomen, podría ser un mecanismo de contagio para el personal de salud. Diversas guías clínicas y trabajos de investigación (nacionales e internacionales) generados en estos últimos meses han logrado rebatir este temor y actualmente el procedimiento laparoscópico nos brinda la oportunidad de tener un mejor control del humo quirúrgico, el cual se encuentra contenido en la cavidad abdominal y puede ser filtrado al momento de evacuarlo ${ }^{(3,4)}$.

Desde otra perspectiva, la pandemia por la COVID-19 ha producido un impacto significativo en los programas de formación médica tanto de pregrado como los de posgrado en especialización (Residentado Médico). Esto, como consecuencia de los cambios producidos en los diferentes escenarios de aprendizaje que se pueden resumir en lo siguiente:

- Suspensión del acceso a los campus universitarios y a los campos clínicos y socio-sanitarios.

- Reconfiguración de la capacidad resolutiva de las instituciones prestadoras de salud (IPRES) dando prioridad a la atención diferenciada en favor de los pacientes con COVID 19.

- Disminución significativa en la atención en áreas de docencia como la consulta externa, cirugía electiva, procedimientos diagnósticos y terapéuticos, entre otros.

- La docencia en servicio se vio desplazada por la actividad asistencial y por lo tanto se suprimieron las actividades académicas hospitalarias tales como visitas, rondas, discusiones de casos clínicos, revisiones bibliográficas.

- Disminución del número de docentes por causa de la propia enfermedad o por ser considerado población vulnerable (edad, comorbilidades).

- Imposibilidad de la movilización tanto nacional como internacional que afectaron los programas de intercambio académico y a las actividades vinculadas a la responsabilidad social universitaria, así como restricciones para participar de eventos académicos internacionales como congresos, simposios, conferencias, etc. 
Este tipo de crisis supone que las facultades de medicina han debido realizar grandes esfuerzos para llevar a cabo planes de contingencia que minimicen las interrupciones o los profundos cambios producidos en los programas educativos de pregrado y del posgrado.

Los programas de residencia se basan en que el médico en formación (residente) desarrolle una actividad directa asistencial supervisada por médicos especialistas que tienen la función de tutores. A esto se le suma las llamadas actividades académicas (discusión de casos, revisión bibliográfica, rondas asistenciales entre otros) que se complementan con actividades de investigación y otras pertinentes a la propia formación de la especialidad. También se incluye dentro del plan de estudios, la rotación en servicios externos a la sede de origen que incluye no solo la atención en establecimientos hasta de segundo nivel sino también la posibilidad de acudir a servicios especializados tanto a nivel nacional como en el extranjero. Todas estas actividades se han visto distorsionadas por las características que demanda la emergencia sanitaria y cuyo efecto ha sido descrito líneas arriba ${ }^{(5)}$

Sin embargo, el panorama para cada especialidad ha sido diferente en términos de afectación de las labores habituales. Es así como, por la naturaleza de la pandemia, los residentes pertenecientes a las especialidades de Medicina Interna, Neumología, Infectología, Cuidados intensivos, Salud Mental, Administración en Salud entre otros, han tenido mayores oportunidades de enfrentar el reto sanitario desde la perspectiva de cada especialidad y probablemente no han tenido mayores contratiempos en cuanto a los contenidos de su programa de formación. En cambio, es distinto el panorama en las especialidades quirúrgicas que son las que más se han visto afectadas dada la restricción de los procedimientos electivos tanto en sala de operaciones, así como el manejo perioperatorio de los pacientes.

En el país, los cronogramas de los programas de residencia no han sido mayormente modificados ni tampoco se consideró la extensión de los mismos por lo cual las brechas existentes en la formación de los residentes, especialmente del área quirúrgica no han sido cubiertas en su totalidad. Por este motivo se tiene plena conciencia de la necesidad de complementar el entrenamiento a estas cohortes que han sufrido el impacto directo por la pandemia de la COVID-19.

La virtualización ha permitido en parte subsanar aspectos cognitivos, pero definitivamente siempre será necesario diseñar programas complementarios que incidan en el desarrollo de destrezas y habilidades para efectuar los diferentes procedimientos quirúrgicos ${ }^{(6)}$. Particularmente en el caso del Hospital Cayetano Heredia, como los residentes de Cirugía no podían acudir al laboratorio de Cirugía Experimental por estar cerradas las instalaciones de la universidad, se decidió trasladar las unidades de entrenamiento en cirugía laparoscópica hacia ambientes del propio hospital lo que permitió que los residentes puedan combinar su labor asistencial con la de entrenamiento sin tener que abandonar su centro hospitalario ${ }^{(1)}$.

En cuanto al pregrado, la decisión del gobierno de turno fue la de suspender la asistencia de los estudiantes a los establecimientos de salud y por ende la participación en cualquier actividad que involucre pacientes y solo se ha permitido el retorno de los alumnos del último año (Internado). Estas medidas inciden fundamentalmente en los estudiantes de años superiores donde su aprendizaje es principalmente en los campos clínicos y socio-sanitarios. Ante esta contingencia, la Facultad de Medicina de la Universidad Peruana Cayetano Heredia ha tenido que diseñar nuevas estrategias de formación que suplan a las actividades tradicionales, teniendo conciencia que la innovación tecnológica ayuda significativamente pero aún no puede reemplazar las experiencias de aprendizaje ante pacientes reales.

En ese sentido se ha utilizado un enfoque basado en competencias para rediseñar las actividades educativas y de evaluación, considerando alternativas al contacto directo con el paciente para cumplir con los resultados de aprendizaje. Si bien los estudiantes de pregrado durante las asignaturas quirúrgicas no participan directamente de los procedimientos en sala de operaciones, se ha restringido el entrenamiento en valoración de los pacientes tanto en el pre como en el postoperatorio, la identificación de probables complicaciones, el manejo de las heridas operatorias, los procedimientos básicos de sutura, entre otros. 
Ante estas restricciones se ha implementado la virtualización en el 100\% de horas de teoría y el mayor número posible de prácticas en laboratorios con el uso de softwares, videos y podcasts tutoriales, simulación clínica virtual, utilizando el contexto de la COVID-19 para la construcción de escenarios. En cuanto a la actividad clínica se está logrando consolidar y adelantar las sesiones didácticas clínicas en línea para permitir un posterior ingreso en el entorno clínico; crear y usar casos virtuales disponibles; modificar el calendario académico para intercambiar experiencias y aplazar las rotaciones clínicas. También se ha dado impulso a la participación de los estudiantes en el entorno de la telesalud, incluyendo rotaciones electivas que les permita participar en equipos de salud como un elemento fundamental para su posterior desarrollo profesional.

La investigación en cirugía ha sido la mejor aliada para entender el comportamiento de la COVID-19 en los pacientes quirúrgicos portadores de esta enfermedad y a la vez nos ha permitido a los cirujanos continuar brindado una atención quirúrgica eficaz y segura tanto para los pacientes como para el personal de salud. Nuestras sociedades quirúrgicas nacionales (Sociedad de Cirujanos Generales del Perú, Sociedad Peruana de Cirugía Endoscópica) se han encargado de difundir lineamientos con este fin a través de diferentes cursos, congresos y reuniones académicas virtuales; asimismo han publicado las experiencias de diversos centros hospitalarios nacionales ${ }^{(1,4,7,8)}$. La colaboración Covid Surg con sede en el Reino Unido ha generado estudios globales con participación de 1667 hospitales en el mundo, provenientes de 116 países; 17 hospitales en el Perú se han sumado a esos estudios colaborativos generando evidencia importante sobre el manejo perioperatorio de los pacientes quirúrgicos en tiempos de pandemia ${ }^{(9)}$.

A dos años de iniciada la pandemia, estamos enfrentando ahora dos fenómenos distintos: por un lado, la llamada ola quirúrgica No Covid que representa el gran volumen de pacientes no atendidos en forma electiva durante el 2020 y el primer semestre del 2021 y que se estuvo volcando a los hospitales buscando un acceso oportuno para la atención de sus enfermedades. Ante esta situación se tuvo que generar diversas estrategias de los departamentos de Cirugía a fin de satisfacer esta gran demanda alentada por los bajos indicadores de incidencia de la COVID-19 en los últimos meses del año 2021. El otro fenómeno que estamos experimentando es el desarrollo de una tercera ola que tiene como protagonista a la variante Omicron pero que aparenta un comportamiento distinto con una mayor contagiosidad, pero menor letalidad lo cual puede suponer que los servicios hospitalarios no se verán comprometidos al extremo como ocurrió en los dos picos previos y que no se altere mayormente la configuración de los mismos ${ }^{(10)}$.

Definitivamente la pandemia de la COVID-19 ha producido un impacto significativo en el área quirúrgica tanto en la labor asistencial, como en la docencia, investigación y la gestión de los servicios quirúrgicos cuyos efectos requieren desarrollar estrategias innovadoras para afrontar estos nuevos retos. La tarea es muy demandante y solo se puede garantizar el éxito sumando esfuerzos desde la perspectiva de cada departamento asistencial, académico, la plana de cirujanos y los propios estudiantes teniendo siempre como propósito fundamental la recuperación de la salud y el bienestar de nuestros pacientes.

Manuel Rodríguez Castro ${ }^{1, \mathrm{a}, \mathrm{b} ; \mathrm{d}}$, Giuliano Borda Luque $\mathrm{e}^{1, \mathrm{c} ; ~ 2, \mathrm{~d}, \mathrm{e} ; \mathrm{f}}$

\footnotetext{
Universidad Peruana Cayetano Heredia. Lima, Perú.

Hospital Cayetano Heredia. Ministerio de Salud. Lima, Perú.

Profesor Principal Facultad de Medicina Alberto Hurtado

Decano de las Facultades de Medicina, Estomatología y Enfermería

Profesor de la Universidad Peruana Cayetano Heredia

Cirujano General

Jefe de Departamento de Cirugía Hospital Cayetano Heredia

Presidente de la Sociedad de Cirujanos Generales del Perú.
} 


\section{REFERENCIAS BIBLIOGRÁFICAS}

1. Acosta H, Cáceres R, Quispe D, Bustamante Z, Borda G. Organización de un Departamento de Cirugía ante el COVID-19. Rev Soc Peru Cir Endosc. 2020; 2(1):28-35.

2. Quijano J, Varillas V, Quispe D, Acosta H, Yamamoto G, Vásquez V, Borda G. Recomendaciones de manejo para pacientes que requieren cirugía de emergencia durante la pandemia COVID-19. Resolución Directoral 136-202-HCHDG. 2020; Disponible en: http://www.hospitalcayetano.gob.pe/PortalWeb/wpcontent/uploads/resoluciones/2020/RD/RD_1362020-HCH-DG.pdf

3. Carpio Y, Cardenas D, Borda G. Emergency Abdominal Laparoscopic Surgery during the Coronavirus Disease 2019 Pandemic: Experience in a Private Center in Peru. J Laparoendosc Adv Surg Tech. 2021; 31(3):261-5.

4. Aliaga S, Anchante E, Chiroque L, Borda G. COVID-19 y Cirugía: Recomendaciones de la Sociedad Peruana de Cirugía Endoscópica. Rev Soc
Peru Cir Endosc. 2020; 2(1):14-20.

5. Nepogodiev D, Omar OM, Glasbey JC, Li E, Simoes JFF, Abbott TEF, et al. Elective surgery cancellations due to the COVID-19 pandemic: global predictive modelling to inform surgical recovery plans. $\mathrm{Br} \mathrm{J}$ Surg. 2020; 107(11):1440-9.

6. Rai O, Fernandes R. COVID-19 and the reintroduction of surgical training. BJS. 2021;108(1):e6.

7. Huaman E, Vergel C, Ugarte R. Apendicectomías en pacientes adultos con infección por SARS-CoV-2 en un hospital especializado en el tratamiento del COVID-19. Rev Soc Peru Cir Endosc. 2020; 2(1):227.

8. Zegarra $\mathrm{S}$, Verástegui $\mathrm{T}$, Auris $\mathrm{H}$. Pacientes con falla intestinal atendidos en tiempos de la pandemia por COVID-19 en la Unidad de Falla Intestinal. Cirujano. 2020; 17(1):22-8.

9. COVIDSurg Collaborative, GlobalSurg Collaborative. SARS-CoV-2 vaccination modelling for safe surgery to save lives: Data from an international prospective cohort study. Br J Surg. 2021 Sep 27; 108(9):10561063. doi: 10.1093/bjs/znab101.

10. Maslo C, Friedland R, Toubkin M, Kama B. Characteristics and outcomes of hospitalized patients in South Africa during the COVID-19 Omicron wave compared with previous waves. JAMA Published online December 30, 2021. doi:10.1001/ jama.2021.24868. 